\title{
High Alert For Cannabinoid Hyperemesis Syndrome: A Case Report
}

Madhur Rathi.'

\begin{abstract}
Background: A 32-year-old Caucasian man presented with intractable nausea, psychogenic vomiting, abdominal pain and compulsive hot-water bathing behaviors following the habitual use of cannabis for years, consistent with the uncommon and frequently overlooked diagnosis of Cannabinoid Hyperemesis Syndrome. This was his third admission to the emergency department with the same complaints and symptoms which had persisted for over two years without a recognizable etiology. All imaging studies done on each visit were unremarkable. Results: The patient was clinically symptomatic with the aforementioned presenting complaints, but disappeared upon discontinuation of the cannabis. Within two days of supportive treatment in addition to temporary relief of symptoms with bathing. To date, no effective cure has been sought for this unique diagnosis other than abstaining from cannabis use. Conclusion: A complete recovery was made three days following admission. The presenting symptoms were attributed to the smoking behaviors. The patient was followed up by his primary care physician once released from the hospital. A very rare diagnosis surfaced a number of times with the same patient in the same setting over the span of a couple years, but was overlooked due to its rarity. Hence, physicians should list it higher on their differentials when dealing with a patient with a history of drug abuse.
\end{abstract}

Keywords: Cannabinoids, Vomiting, Baths, Compulsive Behavior (Source: MeSH, NLM).

About the Author: Madhur Rathi is a fourth year medical student at UMHS. He aspires to pursue a family medicine residency with an emergency medicine fellowship.
Submission: May 6, 2014 Acceptance: Oct 13, 2014 Process: Peer-reviewed

\section{Introduction}

Cannabinoid Hyperemesis Syndrome (CHS) was first noted by Allen $\mathrm{JH}$, and De Moore GM, when they explored the association between chronic cannabis abuse and a cyclical vomiting illness that presented in a series of cases in South Australia in 2004.' It is a syndrome characterized by the chronic use of cannabis, recurrent episodes of severe nausea, vomiting, abdominal pain, and habitual bathing behaviors to help resolve symptoms (a pathognomonic feature). The mechanisms explaining the adverse consequences of long-term cannabis toxicity remain largely unknown, however various pathogenic mechanistic theories maintain that several peripheral $\mathrm{CB}_{1}$ cannabinoid receptors in the brain are responsible for the presenting signs and symptoms.' Almost a decade has passed since the first published case of CHS and yet in the emergency department, this peculiar disease remains obscure and under diagnosed. In fact, before a case series published by simonetto et al., at the Mayo Clinic in 2012 recognizing 98 patients afflicted with the syndrome, only 33 cases of CHS was published. ${ }^{2}$ Since the first aforementioned documentation and with the recent legalization of medical marijuana in numerous states, the syndrome has now surfaced across North America, Western Europe and Australia. $^{3}$

\section{The Case}

A 32 year old male presented to the Dekalb Medical Center Emergency Department in Atlanta, Georgia for nausea, nonbloody vomiting, and diffuse colicky abdominal pain over the course of a week. Food intake exacerbated his condition and

Key Points:
- The low number of reported cases despite the pathognomonic features
of the syndrome render CHS rather difficult to diagnose clinically.
- As cannabinoids are supposed antiemetics, their habitual use leading to
vomiting as one of the more potent side effects is a conundrum.
- The ironic nature of the syndrome has led to theories being proposed
regarding its mechanism, but none of the hypotheses have been confir-
med to date.

his nausea was relieved by taking hot showers. Patient admitted to smoking half a pack of cigarettes per day as well as smoking up to five blunts of cannabis a day for the past six years. The rest of the review of systems was noncontributory. On physical examination, his blood pressure was 140/90, his pulse rate was $104 / \mathrm{min}$ and his mucous membranes were dry. The remainder of the physical examination, his complete blood count, comprehensive metabolic panel and arterial blood gas were all unremarkable. Serum amylase and lipase levels were within therapeutic range. His urine drug screen was positive for tetrahydrocannabinol (THC). It was later noted that these symptoms had persisted for over 2 years and the patient had been admitted with the same complaints three times to the same emergency department. Each time, abdominal X-Rays, CT scans, ultrasounds, esophagogastroduodenoscopies, colonoscopies, and stool studies were performed and were all unremarkable. No previous interventions were noted on each visit. In his third and final admission, the clinical diagnosis of CHS was made. Treatment was supportive with fluid hydration and rest. The patient abstained from cannabis use for the duration of his stay in the hospital until discharge three days later.

${ }^{1}$ University of Medicine and Health Sciences, Faculty of Medicine, Canada. 


\section{Discussion}

It has been noted that It takes up to an average of seven visits before patients with chronic vomiting are diagnosed with any underlying cause. ${ }^{4}$ In fact, in a survey conducted by Venkatesan T, et al., revealed that $89 \%$ of the patients with chronic vomiting were not diagnosed in the emergency department. ${ }^{3}$ Emergency medicine physicians may be hesitant to place the diagnosis of CHS high on their differentials for the following reasons:

CHS is a rare diagnosis: As mentioned previously, up until two years ago, few cases had been published for $\mathrm{CHS}^{2}{ }^{2}$ Although this low number suggests that the diagnosis is uncommon, medical professionals and cannabis users may not be conscious of the diagnostic symptoms. Consequently, a significant number of cases might be unrecognized. Here a case of cannabis use was described where the patient presented multiple times with the same complaints and an exhaustive medical evaluation failed to reveal another etiology. Several common differentials for nausea and vomiting were considered including overdoses of alcohol or medications, bacterial and viral infections, inflammatory gastrointestinal diseases, and central nervous system or psychiatric conditions. However, no evidence supported any of the aforementioned causes.

CHS is a paradoxical diagnosis: Cannabinoids are medically used antiemetics for the prevention of nausea and vomiting caused by chemotherapy via a mechanism inhibiting the release of emetic neurotransmitters by stimulating presynaptic cannabinoid CB1 receptors. ${ }^{5}$ Hence, cannabinoids inducing hyperemesis appears to be contradictory and the pathophysiological mechanism(s) underlying the induced vomiting is still unknown, therefore obscuring the role of cannabinoid in patients with hyperemesis.

CHS is an under-researched diagnosis: The pathognomonic feature of CHS of compulsive warm water bathing for temporary relief of symptoms has no definite explanation. The $\mathrm{CB} 1$ receptor is near the thermoregulatory center of the hypothalamus and chronic stimulation may be counteracted with warm showers, but to date, this theory has yet to be verified. ${ }^{6}$ Alter- nate explanations theorize that $\mathrm{CB} 1$ receptor-mediated vasodilation of the gut with chronic cannabis use may contribute to the symptoms. A redistribution of blood flow from the gut to the skin with hot water could bring relief to the patient in a "cutaneous steal syndrome" pattern. ${ }^{2}$

The aforementioned case series published in 2012 by Simonetto et al., identified 98 patients who met inclusion criteria for diagnosis - recurrent vomiting with no other explanation for symptoms, cannabis use preceding symptom onset and absence of major illness that could explain the symptoms. To further refine the decisive factors, the authors have now expanded the flow chart to include relief of symptoms with hot showers or baths, resolution with cannabis cessation, several cycles of nausea and vomiting, abdominal, epigastric or periumbilical pain, weekly use of marijuana, age under 50 , weight loss greater than $5 \mathrm{~kg}$, normal bowel habits, negative laboratory, radiographic, and endoscopic test results with a morning predominance of presenting symptoms. ${ }^{2}$ These can all further aid ED physicians in formulating their differentials when confronted with patients who present with similar symptoms to rule out differentials such as gastroesophageal reflux disease, peptic ulcer disease, inflammatory bowel disease, appendicitis and pancreatitis among other gastrointestinal diseases high on the possible diagnoses without colonoscopies, endoscopies, esophagogastroduodenoscopies and imaging studies to aid them. Still, studies with lower attrition rates are needed and validation of the proposed diagnostic criteria is required.

\section{Conclusion}

The use of cannabis has become more prominent and it is slowly gaining notoriety as one of the most commonly abused drugs worldwide. In fact, an estimated 2.6 million individuals in the USA become new users every year.' Though further studies are warranted to further understand the prevalence and symptoms of this uncommon disease, CHS should be considered higher on the differential list when patients present with chronic vomiting with no underlying etiology given the widespread and continuously rising use of cannabis today. With an accurate history and knowledge of this phenomenon, expensive and invasive diagnostic treatment can be avoided. 


\section{References}

1. Allen JH, de Moore GM, Heddle R, Twartz JC. Cannabinoid hyperemesis: cyclical hyperemesis in association with chronic cannabis abuse. Gut. 2004 Nov:53(11):1566-70.

2. Simonetto DA, Oxentenko AS, Herman ML, Szostek JH. Cannabinoid hyperemesis: a case series of 98 patients. Mayo Clin Proc. 2012 Feb;87(2):114-9. 3. Venkatesan T, Tarbell S, Adams K, McKanry J, Barribeau T, Beckmann K, et al. A survey of emergency department use in patients with cyclic vomiting syndrome. BMC Emerg Med. 2010 Feb 24;10:4.
4. Darmani NA. Cannabinoid-Induced Hyperemesis: A Conundrum-From Clinical Recognition to Basic Science Mechanisms. Pharmaceuticals. 2011 Jul;3(7):2163-77.

5. Harvey DJ, Brown NK. Comparative in vitro metabolism of the cannabinoids. Pharmacol Biochem Behav. 1991 Nov;40(3):533-40.

6. Sullivan S. Cannabinoid hyperemesis. Can J Gastroenterol. 2010 May;24(5):284-5.

7. Chang YH, Windish DM. Cannabinoid hyperemesis relieved by compulsive bathing. May Clin Proc. 2009;84(1):76-8.

\section{Acknowledgments}

None.

Conflict of Interest Statement at Funding

The author has no funding, financial relationships or conflicts of interest to disclose.

Author Contributions

Conception and design the work/idea, Collect data/obtaining results, Analysis and interpretation of data, Write the manuscript: MR.

Cite as:

Rathi M. High Alert For Cannabinoid Hyperemesis Syndrome: A Case Report. Int J Med Students. 2014 Jul-0ct;2(3):135-7. 